\title{
On-demand Milifluidic Synthesis of Quantum Dots in Digital Droplet Reactors
}

Craig Richardab†, Rachel McGee ${ }^{a \dagger}$, Aditya Goenkac, Prabuddha Mukherjee ${ }^{a}$, and Rohit Bhargavaabde

aBeckman Institute for Advanced Science and Technology, University of Illinois at Urbana Champaign, Urbana, IL 61801, USA

bDepartment of Bioengineering, University of Illinois at Urbana-Champaign, Urbana, IL, 61801, USA

'Department of Chemical Engineering, Indian Institute Technology, Kharagpur, India dDepartments of Bioengineering, Chemical and Biomolecular Engineering, Electrical and Computer Engineering, Mechanical Science and Engineering and Chemistry, University of Illinois at Urbana-Champaign, Urbana, IL 61801, USA

eCancer Center at Illinois

†Authors contributed equally to this work

${ }^{*}$ Corresponding Author

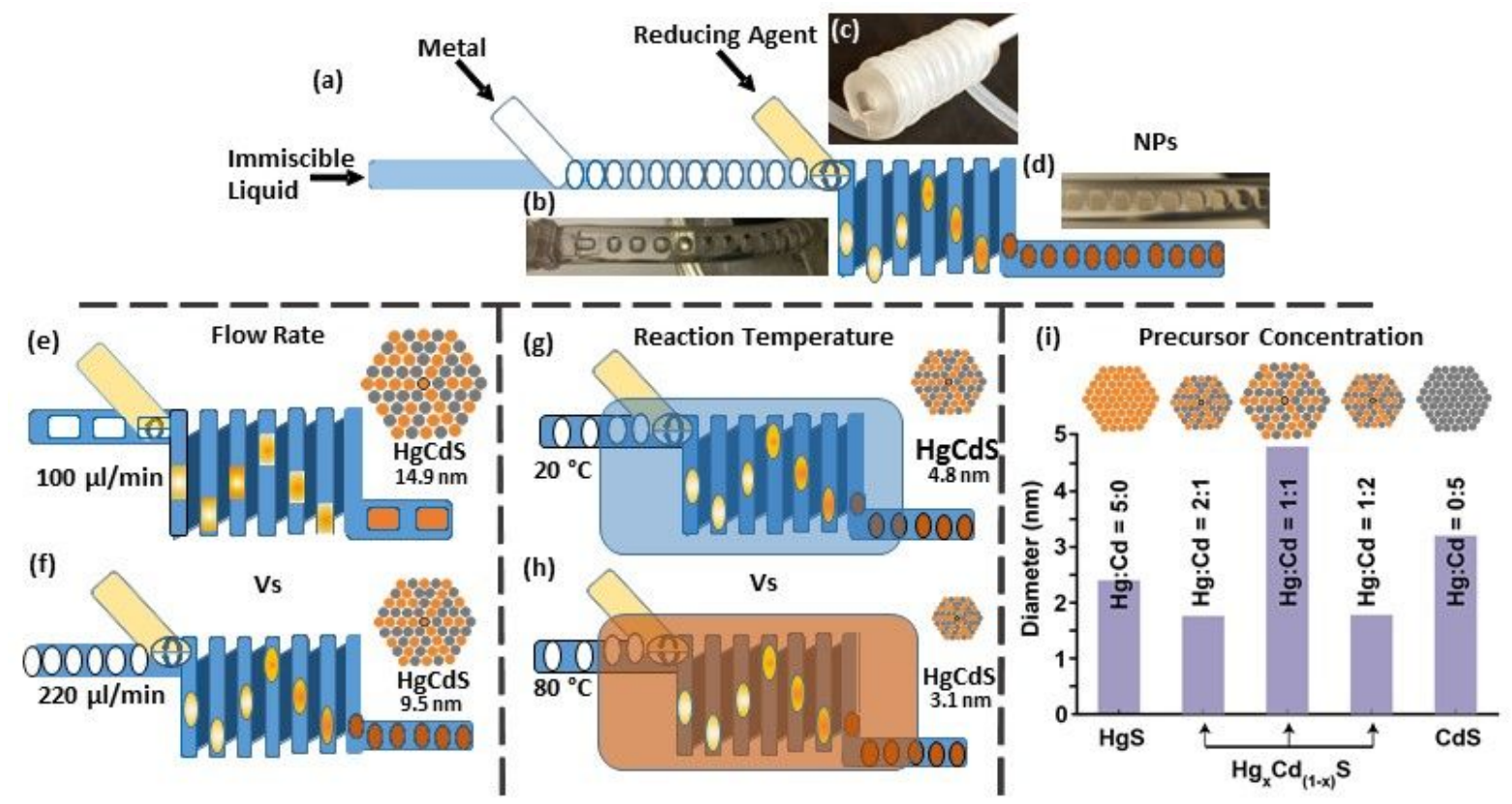

Figure S1:(a) Schematic representation of the droplet reactor microfluidic device,(b) Image of the formation of droplets as the flow of metals (aq) meets a flow of hydrophobic paraffin oil,(c) Image of 3D printed helical mixer,(d) Image of the formation of QDs in the droplet based microfluidic device,(e-f) Demonstrates the effect of flow rate on the reaction vessel and QD size,(g-h) Schematic representation of reaction, immersed in an oil bath, at increasing temperatures and effect on $Q D$ diameter and,(i) Demonstrates the effects of precursor concentration on QD size. 


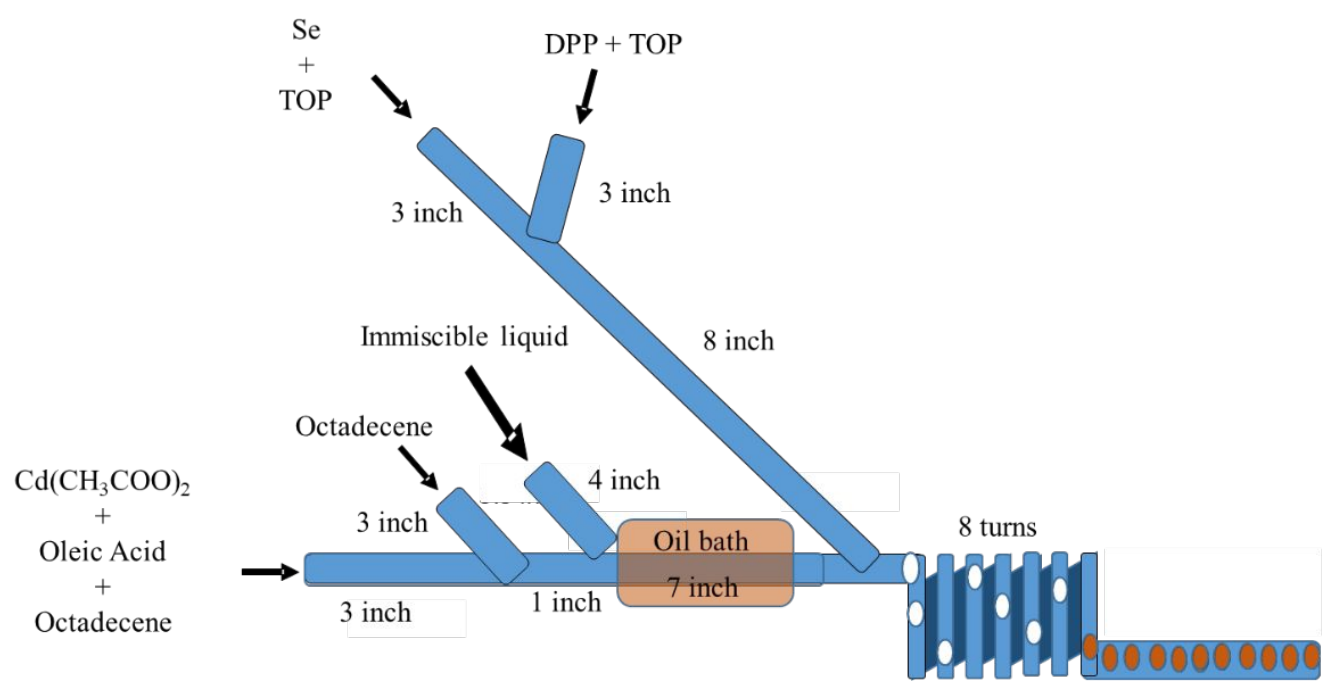

Figure S2: Schematic representation of the organic synthesis droplet reactor with labeled inputs 
Table S1: Demonstrates changes in concentration, temperature and flow rate affecting NP size and subsequently emission maxima. Errors arise from the standard deviation of three measurements from five samples.

\begin{tabular}{|c|c|c|c|c|c|c|}
\hline $\begin{array}{c}\text { Sampl } \\
e\end{array}$ & $\begin{array}{c}\text { Temperatur } \\
\left.\text { e ( }{ }^{\circ} \mathrm{C}\right)\end{array}$ & $\begin{array}{c}\text { Flow } \\
\text { rate } \\
(\mu \mathrm{l} / \mathrm{min} \\
)\end{array}$ & $\begin{array}{c}\text { De } \\
\text { Numbe } \\
r\end{array}$ & $\begin{array}{c}\text { NP } \\
\text { diamete } \\
\text { r by } \\
\text { TEM } \\
(\mathrm{nm})\end{array}$ & $\begin{array}{c}\text { NP } \\
\text { diamete } \\
\text { r by } \\
\text { DLS } \\
(\mathbf{n m})\end{array}$ & $\begin{array}{c}\text { Emissio } \\
n(n m)\end{array}$ \\
\hline 1 & 17 & 100 & 1.30 & $\begin{array}{c}6.45 \pm \\
0.77\end{array}$ & $\begin{array}{c}19.07 \pm \\
0.94\end{array}$ & 784 \\
\hline 2 & 17 & 120 & 1.58 & $\begin{array}{c}6.05 \pm \\
0.69\end{array}$ & $\begin{array}{c}14.90 \pm \\
0.98\end{array}$ & 731 \\
\hline 3 & 17 & 140 & 1.84 & $\begin{array}{c}5.49 \pm \\
0.27\end{array}$ & $\begin{array}{c}11.18 \pm \\
1.02\end{array}$ & 728 \\
\hline 4 & 17 & 160 & 2.10 & $\begin{array}{c}5.36 \pm \\
0.52\end{array}$ & $\begin{array}{c}10.43 \pm \\
1.02\end{array}$ & 720 \\
\hline 5 & 17 & 180 & 2.37 & $\begin{array}{c}5.29 \pm \\
0.83\end{array}$ & $\begin{array}{c}10.20 \pm \\
0.99\end{array}$ & 755 \\
\hline 6 & 17 & 200 & 2.64 & $\begin{array}{c}2.62 \pm \\
0.56\end{array}$ & $\begin{array}{c}9.70 \pm \\
1.26\end{array}$ & 713 \\
\hline 7 & 17 & 220 & 2.90 & $\begin{array}{l}4.32 \pm \\
0.65\end{array}$ & $\begin{array}{c}9.32 \pm \\
1.15\end{array}$ & 712 \\
\hline 8 & 17 & 240 & 3.17 & $\begin{array}{c}4.14 \pm \\
0.27\end{array}$ & $\begin{array}{c}9.01 \pm \\
1.34\end{array}$ & 698 \\
\hline 9 & 17 & 260 & 3.43 & $\begin{array}{c}4.09 \pm \\
0.35\end{array}$ & $\begin{array}{c}8.50 \pm \\
1.58\end{array}$ & 682 \\
\hline 10 & 30 & 180 & 2.97 & $\begin{array}{c}4.90 \pm \\
0.51\end{array}$ & $\begin{array}{c}14.85 \pm \\
0.71\end{array}$ & 729 \\
\hline 11 & 40 & 180 & 3.61 & $\begin{array}{c}4.90 \pm \\
0.24\end{array}$ & $\begin{array}{c}15.62 \pm \\
1.65\end{array}$ & 735 \\
\hline 12 & 50 & 180 & 4.29 & $\begin{array}{c}4.80 \pm \\
0.59\end{array}$ & $\begin{array}{c}13.41 \pm \\
0.98\end{array}$ & 752 \\
\hline 13 & 55 & 180 & 4.62 & $\begin{array}{c}4.46 \pm \\
0.69\end{array}$ & $\begin{array}{c}12.20 \pm \\
1.54\end{array}$ & 806 \\
\hline 14 & 60 & 180 & 5.00 & $\begin{array}{c}4.31 \pm \\
0.24\end{array}$ & $\begin{array}{c}9.90 \pm \\
1.64\end{array}$ & 789 \\
\hline 15 & 65 & 180 & 5.31 & $\begin{array}{c}3.56 \pm \\
0.28\end{array}$ & $\begin{array}{c}8.41 \pm \\
2.00\end{array}$ & 801 \\
\hline
\end{tabular}




\begin{tabular}{|c|c|c|c|c|c|c|}
\hline 16 & 70 & 180 & 5.75 & $\begin{array}{c}3.10 \pm \\
0.27\end{array}$ & $\begin{array}{c}8.23 \pm \\
2.16\end{array}$ & 791 \\
\hline 17 & 80 & 180 & 6.51 & $\begin{array}{c}4.80 \pm \\
0.59\end{array}$ & $\begin{array}{c}8.45 \pm \\
3.64\end{array}$ & 819 \\
\hline
\end{tabular}

Dean number (De) is a dimensionless quantity used to describe the behavior of fluid flow in curved channels and expresses the relative magnitudes of inertial and centrifugal forces to viscous forces. It is equal to Reynolds number multiplied by the square root of the ratio of the diameter of the channel (D) and twice the radius of curvature $\left(R_{\mathrm{c}}\right)$ of the path the channel follows.

Written explicitly $D e=R e \sqrt{\frac{D}{2 R_{c}}}$

Written this way it can be observed that it is a modification of the Reynolds number by the curvature of the channel and is a result of changes in momentum between fluid at the inside and outside of the curved channel/

Effect of flow rate: As demonstrated in Table $S 1$ the synthesis was performed at different $D e$ typical of flow rates in common laboratory conditions $(100 \mu \mathrm{l} / \mathrm{min}$ to $260 \mu \mathrm{l} / \mathrm{min}$ ). Figure S3(a) demonstrates the correlation between the size of QD synthesized and the De number dictated specifically by increasing the flow rate. It was observed that as the De increased, the NP diameter decreased, as measured by transmission electron microscopy (TEM), (Table S1(1-17)). This was to be expected as a faster flow rate provides an increased mixing efficiency, hence smaller uniform NPs would be formed. Dynamic light scattering (DLS) was also performed to ensure that the solution as a whole exhibited this narrow size distribution (Table S1). DLS data obtained showed a decrease in NP diameter with increasing De consistent with TEM data. Furthermore Figure S3(a), gray triangles, demonstrates that the model successfully predicted the QDs size with increasing flow rate. Discrepancies at higher flow rates may be due to the inadequate consideration of local gradients from small imperfections in the device. With increased flow rates, the effects become more prominent and experimental diameters are smaller than predicted. The decrease in QD diameter resulted in a blue-shift, from 763 to $693 \mathrm{~nm}$, in the absorption maxima, with the emission also blue-shifted, from 793 to $549 \mathrm{~nm}$ as shown in Figure S3(c). Interestingly, the peak full width at half maximum height (FWMH) for QDs' photoluminescence emission may be hundreds of $\mathrm{nm}$ due to large size distributions in batch synthesis; ${ }^{1-2}$ however here we observe FWHM of 43, 21, 13 and $19 \mathrm{~nm}$ for QDs synthesized at flow rates of $100,180,220$ and $260 \mu \mathrm{l} / \mathrm{min}$. The narrow emission is likely a direct consequence of the small size distribution obtained from synthesis performed in the device. The relatively narrow emission for a reaction conducted in an automated manner and simple setups provides confidence that the QDs are usable, can be reproducible and this pathway can be utilized for multiplexed fluorescence detection. Both of these characteristics are important for biomedical analyses.

\section{Modelling Nanoparticle Synthesis in the Microfluidic Device:}

To predict the temperature and flow rate that produces NPs of desired size a classical diffusion-based model has been developed. The synthesis of NPs as a sequence of three main steps: Nucleation, growth and ligand capping. Nucleation represents the initial stages during the crystallization process and its rate can be defined as the increase in the number of particles as a function of time according to the following equation: 


$$
\frac{d N}{d t}=\left[\frac{C_{0} k T}{3 \pi \eta d^{3}}\right] \cdot \exp \left(\frac{-16 \pi^{3} V_{m}^{2}}{3 k^{3} T^{3} N_{A}^{2}(\ln S)^{2}}\right)
$$

where, $C_{0}$ is the initial concentration of NPs, $k$ is the Boltzmann constant, $T$ is the temperature of the solution, $\eta$ is the viscosity of the solution, $S$ is its oversaturation and $d$ is the diameter of the growing species. The rate of growth of the NP is dependent on two mechanisms: diffusion of constituents towards the surface on the NPs and surface reaction. ${ }^{3}$ Since we consider the growth of NPs to be diffusion limited, we utilize simplified Fick's law to describe the growth:

$\frac{\mathrm{dr}}{d t}=\frac{D V_{m}}{r}\left(c_{b}-c_{r}(r)\right)$

where, $D$ represents the diffusion coefficient, $V_{m}$ is the molar volume of the solid, $r$ is the particle radius, $c_{b}$ is the bulk concentration of monomers in the solution, and $c_{r}$ is the solubility of the particle with radius $r$. Finally, we account for the hindering of growth due to the ligand binding to the surface of NPs. Ligand capping is modeled mathematically by using Langmuir's adsorption theory in which a fractional area $(\theta)$ of the surface of NPs is used by the ligands and the remaining sites are available for its growth.

$\theta=1-\left(\frac{1}{1+k c t}\right)$

where, $k$ is the Langmuir constant, $c$ is the concentration of ligands and $t$ is time.
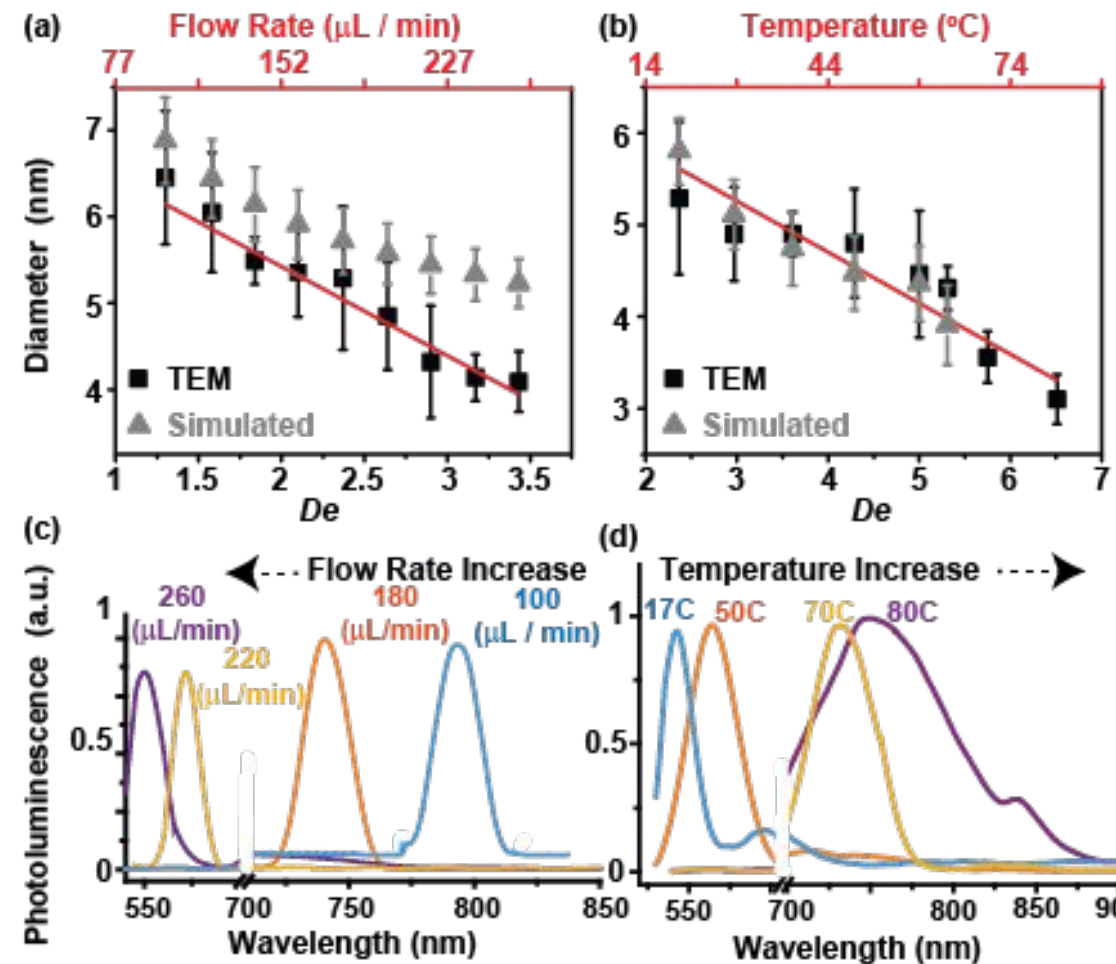

(d)

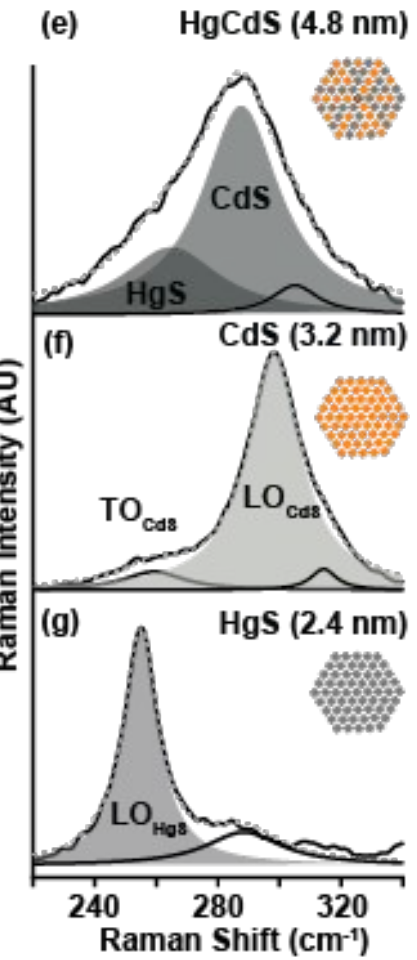

Figure S3:(a) Linear regression (red) demonstrating correlation between QD diameter $(\mathrm{nm})$ and increasing $D e$ number, error (black) arises from standard deviation of 100 QDs measured by TEM per sample. Model predictions (gray triangle) of QD size vs change in flow rate, (b) Linear regression (red) demonstrating correlation between QD 
diameter ( $\mathrm{nm}$ ) and increasing De number, error (black) arises from standard deviation of 100 QDs measured by TEM per sample. Model predictions (gray triangle) of QD size vs change in reaction temperature, (c) Photoluminescence spectra of four $\mathrm{HgCdS}$ QDs synthesized at increasing flow rates (FR): 100 (blue), 180 (red), 220 (yellow) and 260 (purple) $\mu \mathrm{l} / \mathrm{min}$, (d) Photoluminescence spectra of four HgCdS QDs synthesized at increasing reaction temperature (T): 17 (blue), 50 (red), 70 (yellow), and 80 (purple) ${ }^{\circ} \mathrm{C}$. Spectra obtained were excited with a $532 \mathrm{~nm}$ laser excitation for $10 \mathrm{~s}$, (e) Raman phonon modes obtained from HgCdS, (f) CdS only and (g) HgS only. Spectra shown was obtained using $532 \mathrm{~nm}$ laser excitation for $30 \mathrm{~s}$.

Effect of reaction temperature: In addition to flow rate, the effects of increasing the reaction temperature was also investigated. Device temperature was controlled by submerging the helical reactor in a common heated oil bath. As the temperature of the reaction was increased from 17 to $80{ }^{\circ} \mathrm{C}$, the $\mathrm{De}$ increased due to a decrease in kinematic viscosity of the reaction droplet and nucleation and diffusion increased. A correlation between QD size and De number (Figure S3(b)) was observed that was similar to that obtained for the flow rate, emphasizing that control over size is via a simple parameter. Upon increasing the temperature of the reaction, the metal monomers are consumed faster in nucleation, this means that a high concentration of QDs are synthesized. The model developed (Figure S3(b)) predicts the concentration of QDs formed at each time step and subsequently it determines the rate of dissolution. Higher temperatures are desirable for narrow size distribution due a decrease in rate of dissolution, implying there are less free monomers in the initial growth solution. As demonstrated in Table S1, the QDs exhibited a decrease in size from 5.29 to $3.10 \mathrm{~nm}$ upon increase in reaction temperature (Figure S3(b)) within the range here. Interestingly, this decrease in QD diameter resulted in a red-shift in both absorption and emission spectra, as shown in Figure S3(d). As the temperature increased from 17 to $80^{\circ} \mathrm{C}$, the photoluminescence peak shifted from 542 to $749 \mathrm{~nm}$. However, it was observed that when the synthesis was performed at $80^{\circ} \mathrm{C}$ the photoluminescence spectrum had two emission peaks. This broadening of the emission peak has been previously observed and is due to the consumption of monomers very rapidly at high temperatures, ${ }^{4}$ this could be overcome by injecting a solution of fresh monomers into to reaction. Implementation of such strategies can be straightforward in our approach.

Optical properties of QDs: Raman active phonon modes of $\mathrm{HgS}$, CdS and mixed metal QDs, HgCdS, were investigated (Figure S3(e-g)). It was expected that HgS would have independent phonon modes present at around $243 \mathrm{~cm}^{-1}$ with the peak blue shifting on increasing size as the HgS bulk peak occurs around $260 \mathrm{~cm}^{-1}$. Similarly for CdS, we expect to observe a peak around $300 \mathrm{~cm}^{-1}$ and for this to blue shift on increasing size, as previously reported. ${ }^{5-6}$ In Figure S3 (g), HgS was found to have an LO mode present at $254 \mathrm{~cm}^{-1}$ and CdS (Figure S3 (f)) was found to have a LO mode, $297 \mathrm{~cm}^{-1}$ and a TO mode at $247 \mathrm{~cm}^{-1}$. However, the mixed metal, HgCdS (Figure S3 (e)), was observed to have a broad phonon mode at $286 \mathrm{~cm}^{-1}$, in between both individual $\mathrm{HgS}$ and $\mathrm{CdS}$ modes, indicating that the sample was truly a mixed $\mathrm{HgCdS}$ QD. 


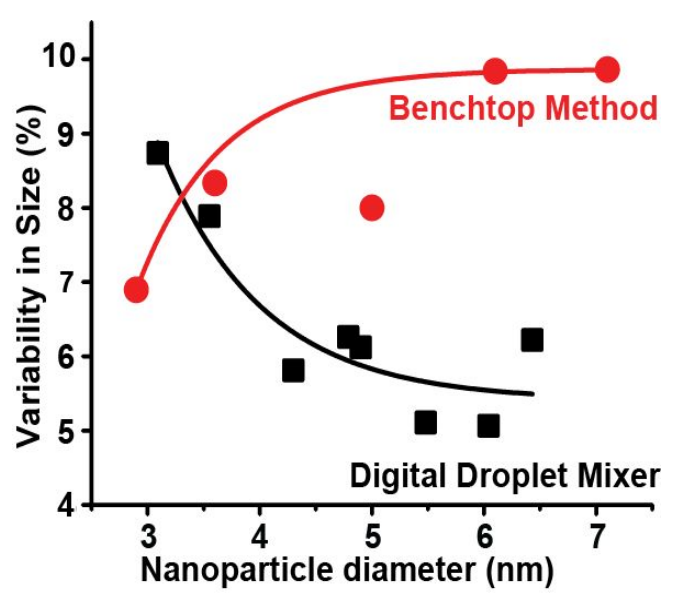

Figure S4: Demonstrates the variability in NP sizes in both a benchtop method vs the variability in size distribution for HgCdS QDs synthesized by our microfluidic device.
Control and reproducibility: Interestingly, the simplicity of this device is a highly effective route of synthesis, that out performs current benchtop methods. Excellent prior work exists in synthesizing a range of $\mathrm{HgS}$ varying in size by increasing the $\mathrm{Hg}: \mathrm{S}$ precursor ratio. ${ }^{7}$ It was observed that as the size increased, the size distribution also increased, as shown in Figure S4 (a). This is to be expected as the reaction for the synthesis of small NPs is fast and hence little mixing is required. However, for larger NPs, the mixing is averaged over a large volume and local variations in conditions may increase variability. In contrast, using the helical microfluidic mixer described, we significantly improve the size distribution of the NPs due to the small reaction volume in the droplet in which conditions are controlled and diffusion is relatively fast and uniform. For smaller NP sizes $(<3.5 \mathrm{~nm})$, we believe that the mixing profile may play a role and the microfluidic device can undergo further optimization. However for larger NPs, up to a twofold reduction in size distribution was obtained, in comparison to the benchtop method. For larger NPs ( $>5 \mathrm{~nm}$ ) we observe a plateau in the size distribution of the NPs at around $5 \%$. Interestingly, this equates to $0.25 \mathrm{~nm}(2.5 \AA)$ which is roughly the size of an $\mathrm{Hg}$ $S$ bond length. Considering NPs are quasi-spherical, a facet is expected to cause variability in size distribution. Therefore, we propose a few $\%$ variation in NP size distribution with our method. Importantly, this is achieved in a simple benchtop scheme that can be set up without significant obstacles. Thus, the approach provides a level of control to researchers that is only available in specialized laboratories at present. 


\section{References}

(1) Cheng, J.; Li, D.; Cheng, T.; Ren, B.; Wang, G.; Li, J. Aqueous synthesis of highfluorescence CdZnTe alloyed quantum dots. J. Alloys Compd. 2014, 589, 539-544.

(2) Bouccara, S.; Sitbon, G.; Fragola, A.; Loriette, V.; Lequeux, N.; Pons, T. Enhancing fluorescence in vivo imaging using inorganic nanoprobes. Curr. Opin. Biotechnol. 2015, 2015 v.34, pp. 65-72.

(3) Shi, J.; Votruba, A. R.; Farokhzad, O. C.; Langer, R. Nanotechnology in drug delivery and tissue engineering: from discovery to applications. Nano Lett. 2010, 10

(9), 3223-30.

(4) Jahn, A.; Reiner, J. E.; Vreeland, W. N.; DeVoe, D. L.; Locascio, L. E.; Gaitan, M. Preparation of nanoparticles by continuous-flow microfluidics. J. Nanopart. Res.

2008, 10 (6), 925-934.

(5) Mukherjee, P.; Lim, S. J.; Wrobel, T. P.; Bhargava, R.; Smith, A. M. Measuring and Predicting the Internal Structure of Semiconductor Nanocrystals through Raman Spectroscopy. J. Am. Chem. Soc. 2016, 138 (34), 10887-10896.

(6) Patel, B. K.; Rath, S.; Sarangi, S. N.; Sahu, S. N. HgS nanoparticles: Structure and optical properties. Appl. Phys. A 2007, 86 (4), 447-450.

(7) Shen, G.; Guyot-Sionnest, P. HgS and HgS/CdS Colloidal Quantum Dots with Infrared Intraband Transitions and Emergence of a Surface Plasmon. The Journal of Physical Chemistry C 2016, 120 (21), 11744-11753. 